\title{
Idosos LGBT que Vivem em Instituições de Cuidados: Desafios e Barreiras para a Manutenção dos Direitos Sexuais
}

\author{
Personas Mayores LGBT que Viven en Instituciones de Cuidado: Desafíos y Barreras para el \\ Mantenimiento de Derechos Sexuales
}

Older LGBT Persons Living in Aged Care Institutions: Challenges and Barriers for Maintaining Sexual Rights

Feliciano Villar

ORCID: http://orcid.org/0000-0003-0830-7034 Universitat de Barcelona, Barcelona/Espanha

Josep Fabà

Universitat de Barcelona, Barcelona/Espanha

Rodrigo Serrat

ORCID: http://orcid.org/0000-0003-3377-681X Universitat de Barcelona, Barcelona/Espanha

Montserrat Celdrán

ORCID: http://orcid.org/0000-0003-3606-1975 Universitat de Barcelona, Barcelona/Espanha

\section{Resumo}

O direito à liberdade de expressão da identidade sexual e orientação de pessoas idosas que vivem em contextos institucionais é submetido a uma série de desafios específicos, que colocá-o em risco. O objetivo deste artigo é identificar e refletir sobre esses desafios e, refletir sobre as medidas para diminui-los ou eliminá-los. Ele descreve o contexto da violência sexual, o estigma associado com o grupo LGTB, e como este pode ser exacerbado entre os idosos. E, em seguida, discute os medos e expectativas das comunidades LGBT no que diz respeito a cuidados institucionais, bem como atitudes para com os membros deste grupo por parte de profissionais e residentes heterossexuais. Por último, várias propostas para garantir os direitos sexuais das pessoas LGBT que vivem em instituições.

Palavras-chaves: Direitos sexuais; Lares para pessoas idosas; As pessoas LGBT; Atitudes.

\section{Resumen}

El derecho a la libre expresión de la identidad y orientación sexual de las personas mayores que viven en entornos institucionales se ve sometido a una serie de desafíos específicos que lo ponen en riesgo. El objetivo del artículo es identificar y reflexionar sobre esos desafíos y reflexionar sobre medidas para atenuarlos o eliminarlos. Para ello se describe el contexto de estigma sexual asociado al colectivo LGTB, y como puede verse agravado entre los mayores. A continuación, se comentan los temores y expectativas que presentan los mayores LGTB respecto al cuidado institucional, así como las actitudes hacia los miembros de ese colectivo tanto de los profesionales como de los residentes heterosexuales. Finalmente, se plantean diversas propuestas para garantizar los derechos sexuales de las personas LGTB que viven en instituciones.

Palabras claves: Derechos sexuales; Residencias de personas mayores; Colectivo LGBT; Actitudes. 


\begin{abstract}
The right to freedom of expression of sexual orientation and identity among older people living in institutional settings is challenged by several specific issues. The aim of the present article is to identity such challenges and discuss about how to remove them or, at least, mitigate their impact. To do so, firstly we describe the context of sexual stigma associated to LGBT people, and how such stigma could be even greater in the case of LGBT older people. Secondly, we discuss LGBT fears and expectation regarding institutional care, as well as attitudes towards such collective held by members of staff and other heterosexual residents. Finally, we pose several proposals to guarantee sexual rights of LGTB people living in long term care institutions for older people.
\end{abstract}

Keywords: Sexual rights; Nursing homes; LGBT persons; Attitudes; Sexual stigma.

\section{Introducción}

El tratamiento de la sexualidad en entornos institucionales donde se ofrecen cuidados a largo plazo para personas ha sido un tema poco estudiado, y sólo en las últimas décadas ha despertado interés tanto desde la investigación como desde la práctica (ver, por ejemplo, Villar, Celdrán, Serrat, Fabà \& Martínez, 2017).

Así, muchos mayores que viven en instituciones siguen manteniendo intereses sexuales y les gustaría poder expresar esas necesidades (Bauer et al., 2013; Hubbard, Tester, \& Downs, 2003). Esta expresión sexual por parte de los residentes, además de no ser un elemento infrecuente dentro de las residencias (Doll, 2013), constituye un derecho que se mantiene en la vejez y que en absoluto prescribe al entrar a vivir en una institución. Los profesionales deberían apoyar este derecho y poner en marcha medidas específicas para garantizarlo, dentro del necesario respeto que también merecen aquellos residentes que prefieren no expresarse sexualmente (Kontos, Grigorovich, Kontos, \& Miller, 2016; Lester, Kohen, Stefanacci, \& Feuerman, 2016).

Sin embargo, la literatura sobre el tema ha destacado algunas barreras con las que se encuentran los mayores que, viviendo en una residencia, desean seguir expresándose sexualmente. Entre estas barreras destaca la alta prevalencia de enfermedades crónicas y de polimedicación que podría inhibir el deseo sexual (Lichtenberg, 2014), la falta intimidad y de espacios lo suficientemente privados como para expresarse sexualmente (Bauer et al., 2013; Morgan, 2009) o, especialmente, las actitudes negativas y prejuicios que mantienen tanto otros residentes (Villar, Celdrán, Fabà, \& Serrat, 2014) como los profesionales que trabajan en residencias (Gilmer, Meyer, Davidson, \& Koziol-McLain, 2010; Hayward, Robertson, \& Knight, 2013).

Obviamente, al hablar de derechos sexuales no nos estamos refiriendo únicamente al comportamiento sexual, sino también a otras facetas de la sexualidad, como la libre expresión de la identidad y orientación sexual. En este sentido, los desafíos y barreras que han de enfrentar las personas lesbianas, gays, bisexuales $o$ transexuales que viven en residencias son particularmente difíciles. El objetivo de la presente revisión es identificar qué desafíos y barreras destacan los estudios de los que ya comenzamos a disponer sobre el tema, lo que nos ofrecerá elementos para reflexionar sobre la mejor forma de ofrecer a las personas mayores no heterosexuales un trato adecuado y no discriminador.

Para ello, estructuraremos nuestro artículo en tres secciones. En primer lugar, contextualizaremos brevemente el tema describiendo qué sabemos sobre las actitudes hacia la sexualidad de las personas mayores LGBT. En un segundo apartado, exploraremos cuáles son las actitudes de las propias personas LGBT ante la expectativa de recibir cuidados institucionales. Seguidamente, señalaremos qué sabemos sobre las actitudes hacia los residentes LGBT que mantienen los 
profesionales que trabajan en instituciones de cuidados a largo plazo, así como de los propios residentes heterosexuales que viven en esas instituciones. Finalmente, concluiremos con una reflexión acerca de lo que necesitamos conocer todavía y cuáles podrían ser las medidas a tomar para respetar los derechos de las personas LGBT que viven en instituciones.

\section{Actitudes hacia las personas mayores LGBT}

Las actitudes sociales hacia las personas LGBT han sido tradicionalmente asociadas al concepto de 'estigma sexual', definido por Herek (2009) como "la visión negativa, el inferior estados $y$ el relativo desempoderamiento que la sociedad colectivamente acuerda otorgar a los comportamientos, identidad, relaciones y comunidad no heterosexual" (p. 66, la traducción es nuestra).

Una vez internalizado, el estigma social da lugar a prejuicios sexuales y a comportamientos discriminatorios en contra de las minorías sexuales, incluyendo entre ellas a las personas LGBT. Esta naturaleza de estas actitudes negativas y su intensidad es susceptible de variar cuando la dimensión sexual se cruza o intersecta con otras dimensiones que también llevan asociado su propio prejuicio. Una de esas dimensiones es la edad. Respecto a la edad, existen números estudios que atestiguan la presencia de actitudes negativas hacia las personas mayores, denominadas edadismo ('ageism', ver Nelson, 2002). De hecho, los estudios sobre edadismo muestran que la sexualidad de las personas mayores ha sido un aspecto tradicionalmente ignorado (en el mejor de los casos) o directamente condenado como algo no natural, sucio o inmoral (Lyons, 2009).

Por desgracia, disponemos de escasos estudios sobre actitudes específicas hacia personas mayores LGBT, aunque recientes avances desde la teoría de la interseccionalidad subrayan la importancia que tiene considerar los efectos (no necesariamente siempre aditivos) de múltiples dimensiones de inequidad que experimentan las personas (Dhamoon, 2011). En este sentido, edad y orientación sexual son en sí mismos dimensiones que general prejuicio, desigualdad $\mathrm{y}$ acceso diferencial a recursos materiales y oportunidades vitales (Calasanti \& Kielcot, 2012; Veenstra, 2011), con lo que es plausible que el estigma asociado a las personas mayores LGBT sea particularmente intenso.

El estigma sexual se expresa tanto desde un punto de vista institucional como individual. Desde el primero de los puntos de vista, es innegable que en las últimas décadas hemos asistido a importantes avances en el reconocimiento de los derechos de las personas LGBT. En numerosos países, particularmente de Europa, América u Oceanía, son ya legales tanto el matrimonio como la adopción de hijos para personas LGBT, y en muchos de ellos existen leyes específicas que otorgan protección legal contra las prácticas discriminatorias basadas en la orientación sexual. Pese a ello, todavía en algunos de esos países los derechos y su expresión práctica no es totalmente igualitaria, así como existen muchos otros (la gran mayoría de países africanos o asiáticos, por ejemplo) en los que las prácticas no heterosexuales o bien no son reconocidas $\mathrm{o}$ bien son oficialmente condenadas y castigadas (Carroll, 2016). Este reconocimiento de derecho probablemente tampoco es igual desde todos los ámbitos institucionales, y hay algunas de ellas, como por ejemplo las instituciones de cuidado, en las que sólo desde hace pocos años se tiene en cuenta la orientación sexual como un elemento a tener en cuenta, considerando el riesgo y especial protección que merecen los derechos de las personas LGBT.

Más allá de estos aspectos institucionales y de la regulación legal, las actitudes individuales hacia las personas LGBT también han variado a lo largo de las últimas décadas. Así, algunos autores hablan de una tendencia hacia la relajación del prejuicio, que sería mucho menor ahora (al menos en algunos países de Europa y América) de lo que lo fue en décadas precedentes (Inglehart, Ponarin, \& Inglehart, 2017). Este cambio, si bien nos hace 
ser optimista, no oculta sin embargo que el prejuicio todavía permanece en muchas personas. Así, la encuestas de opinión de las que disponemos nos hacen ver que incluso en países desarrollados donde la igualdad se ha conseguido desde un punto de vista legal, todavía un importante porcentaje de personas expresan públicamente su antipatía hacia el colectivo LGBT (Adamczyk \& Pitt, 2009; van den Akker, van der Ploeg, \& Scheepers, 2013). Los delitos con motivos ligados a la orientación sexual de la víctima se siguen produciendo y en muchos ámbitos sociales y laborales la personas LGBT siguen siendo discriminadas, lo que lleva a algunos de ellos a no revelar su orientación sexual por miedo a ser discriminadas (Chatterjee, 2014; RodríguezDíaz et al., 2016). Las personas que viven y que trabajan en instituciones de cuidado a largo plazo para personas mayores sin duda no son inmunes a estos prejuicios, y como veremos existen indicios que sugieren que la discriminación subsiste también en esos ámbitos.

Antes de repasarlos, sin embargo, nos gustaría explorar las expectativas que las propias personas LGBT tienen acerca de cómo les gustaría ser cuidados en caso de necesidad y qué temores despierta el contacto con las instituciones y profesionales que se encargarán de ese cuidado en caso de ser necesario.

\section{Actitud de las personas LGBT respecto al cuidado residencial}

Las personas mayores LGBT lo podrían tener especialmente complicado a la hora de llevar una vida sexual coherente con sus deseos en entornos como las residencias, pues a las barreras que mencionamos al principio del presente artículo se le podrían añadir algunas adicionales, incluidas las relacionadas con los prejuicios y estigma sexual que nos referimos en el apartado anterior.

En este sentido, es cierto que las personas mayores heterosexuales y noheterosexuales comparten algunas necesidades, pero también lo es que, en el caso de las segundas, el hecho de pertenecer a una minoría sexual les sitúa en una situación de mayor desventaja (Hughes, Harold, \& Boyer, 2012), especialmente en este tipo de instituciones. De entrada, y éste es un dato importante, las personas mayores homosexuales parecen tener una muy baja predisposición a ir a vivir a una residencia. Según el estudio de McFarland y Sanders (2003), los servicios que podrían resultar más atractivos a este colectivo serían aquellos que les permitieran mantenerse en la comunidad, pues más de la mitad de ellos afirmaron que considerarían la contratación de servicios de atención domiciliaria, servicios de transporte y la posibilidad de vivir en un complejo de apartamentos para personas mayores. En cambio, muy pocas personas se mostraron afines al uso de servicios de larga estancia. De hecho, el 54\% dijeron que no irían a vivir a viviendas tuteladas, el $78 \%$ que no utilizarían un centro de día, y el $81 \%$ que no ingresaría en una residencia.

En otro estudio llevado a cabo por Johnson, Jackson, Arnette y Koffman (2005), se preguntó a un conjunto de personas LGBT de entre 15 y 72 años acerca de sus actitudes sobre las residencias de mayores. En relación a este tipo de instituciones, un $73 \%$ de ellos reportaron creer que la discriminación era un fenómeno real en ellas; un $74 \%$ afirmaron que no creían que la orientación sexual estuviera incluida en sus políticas antidiscriminación, y un $34 \%$ se mostraron convencidos de que se verían forzados a esconder su orientación sexual en el caso de ingresar en uno de estos centros. Aquellos que anticiparon que podrían ser discriminados en las residencias identificaron tres posibles fuentes de discriminación: sus compañeros, el personal administrativo y los cuidadores formales.

También Stein, Beckerman y Sherman (2010), trabajando con una muestra pequeña y metodología cualitativa, comprobaron que había ciertos aspectos que generaban miedo en relación a la provisión de cuidados a largo plazo entre las personas mayores LGBT. Entre estos aspectos se encuentran el rechazo, la negligencia o el abuso por parte de los cuidadores formales, la falta de aceptación y respeto y el maltrato de los compañeros de 
residencia, la posibilidad de tener que ocultar la propia orientación sexual, los sentimientos de soledad por no compartir con otros cuestiones importantes acerca de su identidad, y la falta de servicios gay-friendly.

Los resultados de estos dos estudios no son, para nada, casos aislados que contradigan el resto de literatura científica. De hecho, que las personas mayores LGBT tienen miedo a revelar su orientación sexual a los profesionales de la salud, tanto en entornos institucionales como en la comunidad, es algo que, desgraciadamente, se observa a menudo (ver, por ejemplo, Barbara, Quandt, \& Anderson, 2001; Brotman, Ryan, \& Cormier, 2003; McFarland \& Sanders, 2003; Quam \& Whitford, 1992). Estas preocupaciones podrían explicarse por múltiples motivos, entre los que destacarían la homofobia y el heterosexismo que a menudo que se atribuye a los servicios de salud (Brotman et al., 2003). Así hay, por ejemplo, quien muestra preocupación por la posibilidad de que algunos profesionales sigan considerando la homosexualidad una enfermedad (Barbara et al., 2001). Esto no es de extrañar si se tiene en cuenta que la medicina, entre muchas otras disciplinas, ha defendido este punto de vista durante muchos años y que muchas personas homosexuales que ahora son mayores podrían haber sido sometidas en el pasado a tratamientos para curar su "enfermedad". En otra investigación, Beehler (2001) también encontró a personas gays y lesbianas preocupadas por la posibilidad de ser víctimas de la homofobia de algunos profesionales de la salud, y por el heterosexismo imperante en esta disciplina. Este heterosexismo que se expresaría, por ejemplo, en el hecho que muchos profesionales de la salud, por defecto, dan por supuesto que la persona a la que están atendiendo es heterosexual, lo que genera frustración entre este colectivo (Barbara et al., 2001), y por la atribución a los profesionales de un conocimiento escaso sobre las preocupaciones y problemas de las personas mayores no heterosexuales (McFarland \& Sanders, 2003).

Como acaba de verse, bastantes personas mayores LGBT anticipan la posibilidad de ser discriminadas por parte de los profesionales de la salud, incluidos aquellos que trabajan en residencias que ofrecen cuidados a largo plazo para personas mayores. Éste podría ser uno de los motivos por los que algunas personas no heterosexuales podrían evitar el hecho de acudir a la consulta del médico en busca de cuidados preventivos, atención ginecológica o controles mamarios regulares (Eliason, 1996; Zeidenstein, 1990), lo que puede dificultar, por ejemplo, el diagnóstico precoz de determinadas enfermedades o facilitar su evolución. En otras ocasiones, se decide acudir al médico pero no desvelar la propia orientación sexual, caso en el que el resultado del tratamiento prescrito podría no ser el más adecuado para la persona en cuestión (Grant, 2000).

En cuanto a los servicios de atención y cuidados a largo plazo, se ha observado en este colectivo cierta tendencia a retrasar su uso (Claes \& Moore, 2000), hecho que también podría dificultar la recepción de los cuidados pertinentes en el momento adecuado o el agravamiento de ciertos problemas de salud. En el caso de las residencias, no estar dispuesto a desvelar la propia orientación sexual se puede traducir en verse obligado a hacer una serie de sobresfuerzos para comportarse heteronormativamente, que entre muchas otras cosas implicaría renunciar a llevar una vida coherente con los propios deseos y necesidades. En este tipo de contextos, recordemos, a la preocupación por las posibles reacciones de los profesionales se le suma la preocupación por las del resto de compañeros. ¿Pero son estos recelos realmente justificados? Es decir, ¿profesionales y residentes mantienen actitudes tan negativas hacia la homosexualidad como muchos integrantes del colectivo LGBT piensan?

\section{Actitudes hacia las personas LGBT en entornos institucionales de personas mayores}

En cuanto a las posibles actitudes negativas de los residentes hacia las personas LGBT y su forma real de tratar este colectivo, disponemos de escasos estudios. Sin embargo, 
los pocos disponibles por desgracia avalan que las preocupaciones y miedos de las personas mayores LGBT repasados en el apartado anterior podrían tener una base real. Los estudios principalmente se han dirigido a explorar las actitudes de los residentes, por un parte, y de los profesionales, por otra.

\section{Actitudes de los residentes heterosexuales}

En el caso de los residentes heterosexuales, los escasos estudios de los que se disponen muestran en general actitudes muy prejuiciosas contra sus compañeros del colectivo LGBT.

Por ejemplo, en un estudio en que se planteó un caso hipotético en el que un residente revelase una orientación sexual gay o lesbiana, Villar, Serrat, Fabà y Celdrán (2015a) encontraron que sólo una minoría de residentes en instituciones mostraban reacciones de apoyo o aceptación. Por el contrario, la mayoría de ellos mantenían actitudes negativas, que iban desde un rechazo extremo (un participante comentó: "[pensaría que] es un cerdo asqueroso al que se le vería dar una patada]") hasta otras que indicaban un alejamiento de la persona LGBT. Por ejemplo, una residente mujer indicó:

Muy mal, muy mal... trataría de alejarme de ella [...] trataría de evitarla, y le diría: "No me gusta eso que haces y no me gusta tu actitud. Le pediría por favor que se alejase de mi."

En el mismo estudio, aunque la mayoría de residentes aseguraban que no tendrían problemas compartiendo áreas comunes con residentes gays o lesbianas, también en su mayoría decían que no les gustaría (o directamente, se negarían) a compartir la misma habituación con uno o una de ellos.

Para poder comprender mejor estas actitudes hemos de tener en cuenta el perfil típico de persona mayor que en países como el nuestro, España, ingresa en una residencia. Generalmente son personas que pertenecen a generaciones que no disfrutaron de grandes oportunidades de educación formal, y que además fueron socializadas en valores homofóbicos, según los que una orientación sexual no heterosexual constituía algo delictivo, patológico o pecaminoso. En coherencia con ello podemos mencionar estudios como el de Nieto (1995), quien mostró que las personas mayores parecen dirigir actitudes especialmente negativas hacia la homosexualidad y el lesbianismo. En un mismo sentido apuntan los datos que proporciona la investigación de McIntosh (1981), en la que el $81 \%$ de las mujeres mayores que participaron consideraban inmoral que una persona mayor fuera homosexual (de hecho incluso en algunos casos desconocían el significado de la palabra homosexual), y que Walker, Osgood, Richardson, y Ephross (1998) encontraron que un $60 \%$ de las personas institucionalizadas con las que trabajaron consideraban la homosexualidad algo contra natura, y que un $21 \%$ consideraban que las personas homosexuales no deberían ser admitidas en su institución. Estos datos, que ya tienen algunas décadas, pueden haber mejorado en el presente, pero es probable que sigan mostrando un efecto de la generación por el que los más mayores sean los menos proclives a tener actitudes favorables hacia el colectivo LGBT (Van der Star \& Bänström, 2015). Sin duda necesitamos nuevos estudios que permitan conocer hasta qué punto estas actitudes muestran algún signo de mejora en los últimos años, aunque resultados como los de Villar et al (2015a) no nos permitan ser todo lo optimistas que nos gustaría.

\section{Actitudes de los profesionales}

En relación a las actitudes de los profesionales que trabajan en residencias, la cantidad de evidencia de la que se dispone, aun siendo también escasa, es algo mayor que respecto a las actitudes de los residentes.

Los resultados, sin embargo, no son siempre coincidentes. Por un lado, algunos estudios han encontrado indicios de actitudes prejuiciosas entre los profesionales de la salud y servicios sociales. En el ya citado estudio de 
Walker et al. (1998), no sólo los residentes demostraron tener actitudes negativas hacia la homosexualidad sino que los profesionales también lo hicieron. En esta línea, el 13\% de los profesionales entrevistados se mostraron de acuerdo con que las personas homosexuales no deberían ser admitidas en su centro, y el 55\% de ellos afirmaron que la homosexualidad es algo contra natura. Cuando se ha abordado esta cuestión con profesionales que no se dedicaban específicamente a atender a personas mayores, se han obtenido, también resultados parecidos. Así, otros estudios parecen indicar que un número considerablemente elevado de médicos de familia y psiquiatras experimentan con una frecuencia notable incomodidad o ansiedad a la hora de atender a personas homosexuales, y que hay profesionales de la salud y servicios sociales no están exentos de mantener actitudes prejuiciosas hacia el colectivo LGBT (Dorsen, 2012; Schwinn \& Dinkel, 2015).

En un interesante estudio centrado en residencias de mayores, Hinrichs y VachaHaase (2010) presentaron a 218 profesionales que trabajaban en estos centros una viñeta en la que se narraba una situación en que un profesional descubría a dos personas manteniendo relaciones sexuales, y les preguntaron en qué medida se sentirían sorprendidos e incómodos si se encontraran en esa situación, en qué medida creían que ese comportamiento era aceptable, cómo creían que sería su reacción inicial y en qué medida creían que ignorarían el incidente, intervendrían sobre el mismo o lo reportarían a algún supervisor. Encontraron que la aceptabilidad de esta situación fue significativamente menor en el caso de darse entre personas del mismo sexo (aunque no se observaron diferencias entre el hecho que fueran mujer-mujer u hombre-hombre). En cuanto a sus reacciones iniciales, de nuevo, la positividad de éstas fue significativamente mayor cuando la pareja a la que se descubría manteniendo relaciones era una pareja heterosexual, y los niveles de negatividad no variaron entre la situación mujer-mujer y hombre-hombre. En cuanto a su reacción conductual, la tendencia fue a ignorar la situación y no intervenir en ella, para después reportar lo sucedido a un supervisor. En relación con esta reacción de los profesionales, en algunos estudios en los que se ha preguntado a personas LGBT por la forma como esos profesionales han reaccionado al revelarles su orientación sexual, se reportan algunas respuestas considerablemente negativas (Barbara et al., 2001; Beehler, 2001).

Pero como decíamos, no todas las investigaciones de las que disponemos, sin embargo, llegan a conclusiones tan pesimistas. Estudios como el de Ahrendt, Sprankle, Kuka, y McPherson (2017) o el llevado a cabo por nosotros mismos (Villar, Serrat, Fabà, \& Celdrán, 2015b) han encontrado altas tasas de respeto y aceptación entre los profesionales que trabajan en residencias de personas mayores. Sin embargo, Villar, Serrat, Fabà, y Celdrán (2015b) también han mostrado que la presencia de prácticas de apoyo explícito a las personas LGBT siguen siendo poco comunes entre los profesionales, con el peligro de que los residentes LGBT, sus derechos y sus necesidades específicas puedan pasar desapercibidas en ese tipo de contextos (ver también Neville, Adams, Bellamy, Boyd, \& George, 2015).

\section{Conclusiones}

La evidencia disponible y repasada en el presente artículo nos hace ver la existencia, entre el colectivo LGBT, de una expectativa o temor a convertirse en el blanco de las actitudes y prácticas negativas de los profesionales de la salud. En el caso de residencias de mayores, estos temores incluyen también cierta preocupación ante la posibilidad de tener que convivir con otras personas que puedan discriminarles. Lo más alarmante, no obstante, es que estos temores podrían tener razón de ser, y que de acuerdo a los resultados existentes, la presencia de actitudes y prácticas discriminatorias hacia los residentes LGBT alcanza no sólo a muchos de sus compañeros, sino también a algunos profesionales. 
Las consecuencias de esta situación para los residentes LGBT son claras: gran parte de ellos escogen 'volver al armario' cuando ingresan en una residencia por temor a ser discriminados. Es decir, quizá muchas décadas de vivir su identidad y orientación sexual de manera abierta en la comunidad, entrar en la residencia es un paso atrás dramático y un recorte drástico de derechos (Cohen, Curry, Jenkins Walker, \& Hogstel, 2008; Johnson et al., 2005). El temor a revelar la identidad sexual no sólo tiene efectos en el ámbito de los derechos, sino que puede implicar consecuencias negativas para el funcionamiento psicológico y la salud mental (Beals, Peplau, \& Gable, 2009; Bybee, Sullivan, Zielonka, \& Moes, 2009). Adicionalmente, 'volver al armario' también puede suponer reforzar ciertos sentimientos de aislamiento que muchas veces acompañan a la institucionalización, así como disponer de un menor apoyo social, en cantidad y calidad (Stein, Beckerman, \& Sherman, 2010).

En este sentido, las actitudes de los profesionales que trabajan en residencias es particularmente importante. Hemos de tener en cuenta que no solo son ellos quien proporcionan cuidados directos, sino que son también esos profesionales los responsables de diseñar las normas y políticas que caracterizan a la institución, y de tomar decisiones que afectan a la convivencia en ellas. Debido a esto, su implicación nos parece esencial para que la diversidad sexual dentro de las residencias sea contemplada y se respeten los derechos de los residentes LGBT, eliminado cualquier vestigio de actitudes prejuiciosas o práctica discriminaría tanto en los profesionales como en entre los residentes (Hillman, 2017). Así, son ellos los primeros responsables de asegurar unos cuidados de alta calidad y respetuosos con los derechos de los residentes (Donaldson \& Vacha-Haase, 2016; Porter \& Krinsky, 2014).

Para asegurar que este papel se lleva a cabo adecuadamente, a nuestro juicio se debería incidir en la formación de este colectivo de profesionales en cuestiones relativas a la expresión y derechos sexuales de las personas mayores, incluidas cuestiones que tengan que ver con el colectivo LGBT. Este aspecto de la formación ha sido tradicionalmente olvidado, y es una de las más importantes carencias de los profesionales que trabajan en residencias (Bell, Bern-Klug, Kramer, \& Saunders, 2010). Como resultados, gran parte de ellos no se sienten con las preparados o con las competencias necesarias para ofrecer cuidados adecuados a personas mayores LGBT (Neville et al., 2015). La importancia de la formación lógicamente no se agota en los profesionales, sino que también se debería considerar en el caso de los residentes o incluso de sus familias. En este sentido, tratar de desactivar prejuicios profundamente arraigados durante décadas es una tarea difícil, pero sin duda necesaria si queremos garantizar el buen trato y el sentimiento de inclusión de los mayores LGBT que viven en entornos residenciales.

Estos programas de formación se deberían complementar con políticas institucionales explícitas de apoyo a la diversidad sexual, aceptación de orientaciones sexuales no heterosexuales y detección y erradicación de prácticas discriminatorias en materia sexual. La discusión de si tiene sentido la creación de servicios específicos para personas mayores del colectivo LGBT es una opción planteada desde diversos ámbitos. Ciertos estudios ponen de relieve, por ejemplo, que un porcentaje considerablemente de personas LGBT verían con buenos ojos la creación de servicios gay-friendly (Johnson et al., 2005; Stein et al., 2010), aunque en el estudio de McFarland y Sanders (2003), sólo un $3 \%$ de los participantes indicaron que consideraban importante la creación de servicios dedicados exclusivamente al público LGBT. Es un tema que está abierto a discusión: mientras la adaptación del servicio a las necesidades de ese colectivo, el sentimiento pleno de inclusión y la garantía de sus derechos podrían sugerir que, al menos mientras persista la situación actual, estos servicios tienen sentido, por otro lado se esgrime que la creación de residencias e instituciones de cuidado exclusivas para personas LGBT podría reforzar la segregación de este colectivo. 
Adamczyk, A., \& Pitt, C. (2009). Shaping attitudes about homosexuality: The role of religion and cultural context. Social Science Research, 38(2), 338-351.

Ahrendt, A., Sprankle, E., Kuka, A., \& McPherson, K. (2017). Staff member reactions to same-gender, resident-toresident sexual behavior within Long-Term Care Facilities. Journal of Homosexuality, 64, 1502-1518.

Barbara, A.M., Quandt, S.A., \& Anderson, R.T. (2001). Experiences of lesbians in the health care environment. Women \& Health, 34(1), 45-62.

Bauer, M., Featherstonhaugh, D., Tarzia, L., Nay, R., Wellman, D. \& Beattie, E. (2013). 'I always look under the bed for a man'. Needs and barriers to the expression of sexuality in residential aged care: the views of residents with and without dementia. Psychology and Sexuality, 4(3), 296-309.

Bybee, J. A., Sullivan, E. L., Zielonka, E., \& Moes, E. (2009). Are gay men in worse mental health than heterosexual men? The role of age, shame and guilt, and comingout. Journal of Adult Development, 16(3), 144-154.

Beals, K. P., Peplau, L. A., \& Gable, S. L. (2009). Stigma management and wellbeing: The role of perceived social support, emotional processing, and suppression. Personality and Social Psychology Bulletin, 35(7), 867-879.

Beehler, G. P. (2001). Confronting the culture of medicine: Gay men's experiences with primary care physicians. Journal of the Gay and Lesbian Medical Association, 5(4), 135-141.

Bell, S. A., Bern-Klug, M., Kramer, K. W., \& Saunders, J. B. (2010). Most nursing home social service directors lack training in working with lesbian, gay, and bisexual residents. Social Work in Health Care, 49(9), 814-831.

Brotman, S., Ryan, B., y Cormier, R. (2003). The health and social service needs of gay and lesbian elders and their families in

\section{Referencias}

Canada. The Gerontologist, 43(2), 192-202.

Calasanti, T., \& Kielcot, K. (2012).

Intersectionality and aging families. In R.

Blieszner, \& V. Bedford (Eds.), Handbook

of Families and Aging (pp. 398-434). Santa

Barbara, CA: Praeger.

Carroll, A. (2016). State-sponsored

homophobia: A world survey of sexual

orientation laws: criminalisation, protection and recognition. Geneva:

International Lesbian, Gay, Bisexual, Trans and Intersex Association (ILGA).

Chatterjee, S. (2014). Problems faced by LGBT people in the mainstream society: Some recommendations. International Journal of Interdisciplinary and Multidisciplinary Studies, 1(5), 317-331.

Claes, J. A., \& Moore, W. (2000). Issues confronting lesbian and gay elders: The challenge for health and human services providers. Journal of Health and Human Services Administration, 23(2), 181-202.

Cohen, H. L., Curry, L. C., Jenkins, D., Walker, C. A., \& Hogstel, M. O. (2008). Older lesbians and gay men: Long-term care issues. Annals of Long Term Care, 16(2), 33 .

Dhamoon, R.K. (2011). Considerations on mainstreaming intersectionality. Political Research Quarterly, 64, 230-243. doi: 10.1177/1065912910379227.

Doll, G. M. (2013). Sexuality in nursing homes: Practice and policy. Journal of Gerontological Nursing, 39(7), 30-37.

Donaldson, W. V., \& Vacha-Haase, T. (2016). Exploring staff clinical knowledge and practice with LGBT residents in long-term care: A grounded theory of cultural competency and training needs. Clinical Gerontologist, 39(5), 389-409.

Dorsen, C. (2012). An integrative review of nurse attitudes towards lesbian, gay, bisexual, and transgender patients. Canadian Journal of Nursing Research, 44(3), 18-43.

Eliason, M. J. (1996). Caring for the lesbian, gay, or bisexual patient: Issues for critical 
care nurses. Critical Care Nursing

Quarterly, 19(1), 65-72.

Gilmer, M. J., Meyer, A., Davidson, J., \& Koziol-McLain, J. (2010). Staff beliefs about sexuality in aged residential care. Nursing Praxis in New Zealand, 26(3), 1724.

Grant, J. M. (2000). Outing age: Public policy issues affecting lesbian, gay, bisexual and transgender elders. Wahington, DC: The National Gay and Lesbian Task Force Policy Institute.

Hayward, L. E., Robertson, N., \& Knight, C. (2013). Inappropriate sexual behaviour and dementia: An exploration of staff experiences. Dementia, 12(4), 463-480.

Herek, G.M. (2009). Sexual stigma and sexual prejudice in the United States: a conceptual framework. In D.A. Hope (Ed.),

Contemporary Perspectives on

Lesbian, Gay and Bisexual Identities: The 54thNebraska Symposium on Motivation (pp. 65-111). New York: Springer.

Hillman, J. (2017). The sexuality and sexual health of LGBT elders. Annual Review of Gerontology and Geriatrics, 37, 13-26.

Hinrichs, K. L. M., \& Vacha-Haase, T. (2010). Staff perceptions of same-gender sexual contacts in long-term care facilities. Journal of Homosexuality, 57(6), 776-789.

Hubbard, G., Tester, S. \& Downs, M. (2003). Meaningful social interactions between older people in institutional care settings. Ageing \& Society, 23(1), 99-114.

Hughes, A. K., Harold, R. D., \& Boyer, J. M. (2011). Awareness of LGBT aging issues among aging services network providers. Journal of Gerontological Social Work, 54(7), 659-677.

Inglehart, R.F., Ponarin, E., \& Inglehart, R. C. (2017). Cultural change, slow and fast: The distinctive trajectory of norms governing gender equality and sexual orientation. Social Forces, 95(4), 1313-1340.

Johnson, M. J., Jackson, N. C., Arnette, J. K., \& Koffman, S. D. (2005). Gay and lesbian perceptions of discrimination in retirement care facilities. Journal of Homosexuality, 49(2), 83-102.
Kontos, P., Grigorovich, A., Kontos, A. P., \& Miller, K. L. (2016). Citizenship, human rights, and dementia: Towards a new embodied relational ethic of sexuality. Dementia, 15(3), 315-329.

Lester, P.E., Kohen, I., Stefanacci, R.G., \& Feuerman, M. (2016). Sex in nursing homes: A survey of nursing home policies governing sexual activity. Journal of the American Medical Directors Association, 17(1), 71-74.

Lichtenberg, P. A. (2014). Sexuality and physical intimacy in long-term care. Occupational Therapy in Health Care, 28(1), 42-50.

Lyons, I. (2009). Public perceptions of older people and ageing: A literature review. Dublin: National Centre for the Protection of Older People. Retrieved from http://www.ncpop.ie/userfiles/file/ncpop\%2 Oreports/Review\%201\%20LR\%20Older\%2 0people\%20and\%20ageing.pdf.

McFarland, P. L., \& Sanders, S. (2003). A pilot study about the needs of older gays and lesbians. Journal of Gerontological Social Work, 40(3), 67-80.

McIntosh, D. (1981). Sexual attitudes in a group of older women. Issues in Mental Health Nursing, 3(1-2), 109-122.

Morgan, L.A. (2009). Balancing safety and privacy: the case of room locks in assisted living. Journal of Housing for the Elderly, 23(3), 185-203.

Nelson, T.D. (2002). Ageism. Stereoryping and prejudice against older persons. Cambridge, MA: The MIT Press.

Neville, S. J., Adams, J., Bellamy, G., Boyd, M., \& George, N. (2015). Perceptions towards lesbian, gay and bisexual people in residential care facilities: A qualitative study. International Journal of Older People Nursing, 10(1), 73-81.

Nieto, J. A. (1995). La sexualidad de las personas mayores en España. Madrid: Ministerio de Asuntos Sociales.

Porter, K. E., \& Krinsky, L. (2014). Do LGBT aging trainings effectuate positive change in mainstream elder service providers? Journal of Homosexuality, 61(1), 197-216. Quam, J. K., \& Whitford, G. S. (1992). 
Adaptation and age-related expectations of older gay and lesbian adults. The Gerontologist, 32(3), 367-374.

Rodríguez-Díaz, C.E., Martínez-Vélez, J.J., Jovet-Toledo, G.G., Vélez-Vega, C.M., Hernández-Otero, N.O., Escotto-Morales, B.M., \& Mulinelli-Rodríguez, J.J. (2016). Challenges for the well-being of and health equity of gay, lesbian, bisexual and trans people in Puerto Rico. International Journal of Sexual Health, 28(4), 286-295.

Schwinn, S., \& Dinkel, S. (2015). Changing the culture of long-term care: Combating heterosexism. OJIN: The Online Journal of Issues in Nursing, 20(2).

Stein, G. L., Beckerman, N. L., \& Sherman, P. A. (2010). Lesbian and gay elders and longterm care: Identifying the unique psychosocial perspectives and challenges. Journal of Gerontological Social Work, 53(5), 421-435.

Van den Akker, H., van der Ploeg, R. \& Scheepers, P. (2013). Disapproval of homosexuality: Comparative research on individual and national determinants of disapproval of homosexuality in 20 European countries. International Journal of Public Opinion Research, 25, 64-86. doi:10.1093/ijpor/edr058

Van der Star, A., \& Bränström, R. (2015). Acceptance of sexual minorities, discrimination, social capital and health and well-being: a cross-European study among members of same-sex and opposite-sex couples. BMC Public Health, 15, 812. doi: 10.1186/s12889-015-2148-9.

Veenstra, G. (2011). Race, gender, class, and sexual orientation: intersecting axes of inequality and self-rated health in Canada. International Journal for Equity in Health, 10, 1-11. doi: 10.1186/1475-9276-10-3.

Villar, F., Celdrán, M., Fabà, J., \& Serrat, R. (2014). Barriers to sexual expression in residential aged care facilities: Comparison of staff and residents' views. Journal of Advanced Nursing, 70(11), 2518-2527.

Villar, F., Celdrán, M., Serrat, R., Fabà, J., \& Martínez, T. (2017). Sexualidad en entornos residenciales de personas mayores. Madrid: Fundación Pilares. Recuperado el 8 de julio de 2018 de http://www.fundacionpilares.org/docs/publi caciones/fpilares-guia03-guia-sexualidad2017.pdf.

Villar, F., Serrat, R., Fabà, J., \& Celdrán, M. (2015a). As long as they keep away from me: Attitudes toward non-heterosexual sexual orientation among residents living in Spanish residential aged care facilities (RACFs). The Gerontologist, 55(6), 10061014.

Villar, F., Serrat, R., Fabà, J., \& Celdrán, M. (2015b). Professionals' reactions towards lesbian, gay or bisexual (LGB) people living in residential aged care facilities (RACFs) who actively disclose their sexual orientation. Journal of Homosexuality, 62(8), 1126-1143.

Walker, B. L., Osgood, N. J., Richardson, J. P., \& Ephross, P. H. (1998). Staff and elderly knowledge and attitudes toward elderly sexuality. Educational Gerontology, 24(5), 471-489.

Zeidenstein, L. (1990). Gynecological and childbearing needs of lesbians. Journal of Nurse Midwifery, 35(1), 10-18. 


\section{Dados sobre os autores:}

- Feliciano Villar: Profesor Titular de Universidad en el departamento de Psicología Evolutiva y de la Educación de la Universidad de Barcelona. Doctor en Psicología por la Universidad de Barcelona. Director del Máster Oficial Interuniversitario en Psicogerontología de la Universidad de Barcelona y coordinador del Grupo de Investigación em Gerontología en la citada universidad.

- Josep Fabà: Profesor Asociado en el Departamento de Cognición, Desarrollo y Psicología de la Educación de la Universidad de Barcelona. Doctor en Psicología por la Unviersidad de Valencia. Compatibiliza su trabajo en la universidad con la práctica profesional como psicólogo en una residencia de personas mayores.

- Rodrigo Serrat: Becario Postdoctoral en el Departamento de Cognición, Desarrollo y Psicología de la Educación de la Universidad de Barcelona. Doctor en Psicología por la Universidad de Barcelona. Miembro del Grupo de Investigación em Gerontología y profesor del Máster Oficial Interuniversitario em Psicogerontología de la Universidad de Barcelona.

- Montserrat Celdrán: Profesora Agregada en el Departamento de Cognición, Desarrollo y Psicología de la Educación de la Universidad de Barcelona. Doctora en Psicología por la Universidad de Barcelona. Miembro del Grupo de Investigación em Gerontología y profesora del Máster Oficial Interuniversitario em Psicogerontología de la Universidad de Barcelona. 